\title{
MRI and neurophysiology in vestibular paroxysmia: contradiction and correlation
}

\author{
Christoph Best, ${ }^{1,2}$ Joachim Gawehn, ${ }^{3}$ Heidrun H Krämer, ${ }^{4}$ Frank Thömke, ${ }^{2}$ \\ Tugba Ibis, ${ }^{2}$ Wibke Müller-Forell, ${ }^{3}$ Marianne Dieterich ${ }^{5,6,7}$
}

${ }^{1}$ Department of Neurology, Vestibular Research Unit, Philipps-University, Marburg, Germany

${ }^{2}$ Department of Neurology, Johannes Gutenberg-University, Mainz, Germany

${ }^{3}$ Department of Neuroradiology, University Medical Center, Johannes Gutenberg-University, Mainz, Germany

${ }^{4}$ Department of Neurology, Justus Liebig University, Giessen, Germany

${ }^{5}$ Department of Neurology, Ludwig-Maximilians-University,

Munich, Germany

${ }^{6}$ Center for Vertigo and

Balance Disorders-IFB LMU Ludwig-Maximilians-University,

Munich, Germany

${ }^{7}$ Munich Cluster for Systems

Neurology (SyNergy), Ludwig-

Maximilians-University,

Munich, Germany

\section{Correspondence to}

Dr Christoph Best, Department of Neurology, Vestibular Research Unit, PhilippsUniversity, Baldingerstrasse, Marburg 35043, Germany; bestc@uni-marburg.de

Received 2 April 2013 Revised 10 August 2013 Accepted 14 August 2013 Published Online First 4 September 2013
To cite: Best C, Gawehn J, Krämer HH, et al. J Neurol Neurosurg Psychiatry 2013;84:1349-1356.

\begin{abstract}
Background Vestibular paroxysmia (VP) is defined as neurovascular compression (NVC) syndrome of the eighth cranial nerve (N.VIII). The aim was to assess the sensitivity and specificity of MRI and the significance of audiovestibular testing in the diagnosis of VP.

Methods 20 VP patients and, for control, 20 subjects with trigeminal neuralgia (TN) were included and underwent MRI (constructive interference in steady-state, time-of-flight MR angiography) for detection of a NVC between N.VIII and vessels. All VP patients received detailed audiovestibular testing.
\end{abstract}

Results A NVC of N.VIII could be detected in all VP patients rendering a sensitivity of $100 \%$ and a specificity of $65 \%$ for the diagnosis of VP by MRI. Distance between brain stem and compressing vessels varied between 0.0 and $10.2 \mathrm{~mm}$. In 15 cases, the compressing vessel was the anterior inferior cerebellar artery $(75 \%$, AICA), the posterior inferior cerebellar artery in one $(5 \%$, posterior inferior cerebellar artery $(\mathrm{PICA}))$, a vein in two $(10 \%)$ and the vertebral artery $(10 \%, V A)$ in another two cases. Audiovestibular testing revealed normal results in five patients $(25 \%)$, a clear unilateral loss of audiovestibular function in nine patients $(45 \%)$ and audiovestibular results with coinstantaneous signs of reduced and increased function within the same nerve in six patients (30\%). From the $20 \mathrm{TN}$ patients 7 , (35\%) showed a NVC of the N.VIII (5 AICA, 1 PICA, 1 vein).

Conclusions Only the combination of clinical examination, neurophysiological and imaging techniques is capable of (1) defining the affected side of a NVC and to (2) differentiate between a deficit syndrome and increased excitability in VP.

\section{INTRODUCTION}

Vestibular paroxysmia (VP) is a rare episodic peripheral vestibular disorder leading to acute short attacks of vertigo or disequilibrium with or without auditory and vegetative symptoms. ${ }^{1-3}$ The variability of symptoms is caused by the fact that the eighth cranial nerve (N.VIII) contains different portions: the superior and inferior vestibular nerve and the cochlear nerve. The proposed mechanism underlying the acute attacks is ephaptic depolarisation. Repetitive pathological pressure applied to the nerve by a blood vessel is thought to induce demyelination with succeeding hyperexcitability enabling the ephaptic depolarisation. ${ }^{4-6}$ Thus, the vessels appear to be most often arteries; however, evidence was presented that neurovascular compression
(NVC) syndromes can also be elicited by venous vessels.

Which part of the nerve could be most sensitive to NVC? Anatomically, the course of a cranial nerve is divided into a peripheral and a central part. The part of the cranial nerve between the point of entry (PE: point of entry for afferent fibres, point of exit for efferent fibres) and the area where the nerve is myelinated by Schwann cells is defined as root-entry-zone (REZ). The REZ is histologically myelinated by oligodendroglia. Within the REZ, the susceptibility for developing a NVC syndrome seems to be highest. ${ }^{8}$

In NVC syndromes of other cranial nerves $(\mathrm{N}$. IV; N.V; N.VII) the pathological side due to typical symptomatology can be easily assessed. ${ }^{9-14}$ This, however, is not the case in NVC syndromes of N. VIII, especially when vertigo is the leading symptom and auditory symptoms are lacking. Although, analyses of audiovestibular function in former studies described high rates of pathological test results, a correlation of the side of neurophysiological pathology and imaging results was described as being contradictory. ${ }^{1-3}{ }^{15}$ Up to now, a systematic and detailed evaluation of highresolution MRI in combination with audiovestibular testing is missing in patients with VP. Therefore, the aim of the current study was to present standardised analyses of high-resolution MRI examinations in patients with VP which enable us to define the exact site of a NVC, and compare these to a control group of patients with trigeminal neuralgia (TN). Furthermore, sensitivity and specificity of the MRI procedures were determined, comparing the MRI results with the established clinical diagnostic criteria. Finally, detailed neurophysiological testing of audiovestibular function was correlated to the MRI data in order to define the pathological side.

\section{METHODS}

Inclusion and diagnostic criteria

VP was diagnosed according to the clinical criteria of Brandt and Dieterich. ${ }^{1-3}$ Patients with 'definite' VP as defined by the recently validated diagnostic criteria (see box) without additional criteria concerning NVC with MRI (category E of the diagnostic criteria) were included. MRI data of patients with TN, who underwent the same diagnostic imaging sequences, were used as controls. The study was performed in accordance with the Declaration of Helsinki and after approval of the local ethics committee of the medical association of 
Box Clinical diagnostic criteria for vestibular paroxysmia as adapted from Hüfner and colleagues. ${ }^{3}$

\section{Definite vestibular paroxysmia}

Patients suffered from a minimum of five vertigo attacks and fulfilled one criterion of each category (A)-(E)

- (A) Vertigo attacks: Vertigo attacks (rotatory as well as to and fro vertigo) with short duration (seconds to minutes), which ceased spontaneously

- (B) Vertigo triggers: Attacks occurred while in rest, were induced by a specific head and/or body position, or by a specific change of head and/or body position

- (C) Accompanying symptoms: Attacks were accompanied by at least one of the following additional symptoms: unsteadiness of stance and/or gait, lateralised tinnitus, decreased hearing function or subjective sensory irritations, such as a feeling of pressure within or around one ear

- (D) Additional criteria: The diagnostic procedures in addition to the anamnestic aspects revealed a neurovascular compression of the eighth cranial nerve on MRI scans including CISS sequences, a hyperventilation-induced nystagmus, a detectable progress of vestibular deficit over the course of disease, or patients responded positive to treatment

- (E) Exclusion: Any other possible pathology or disease explaining the symptoms had to be excluded

Probable vestibular paroxysmia

Patients suffered from a minimum of five vertigo attacks and fulfilled criterion (A) and additionally at least three criteria out of the categories (B)-(E)

CISS, constructive interference in steady state.

Rhineland-Palatinate. Patients were included after they gave their informed, written consent.

\section{MRI protocol}

The study was performed using a standardised MRI protocol. All structural images were acquired with the patients positioned supine within a clinical $1.5 \mathrm{~T}$ scanner (Vision Magnetom, Siemens, Erlangen, Germany). The heads of the patients were placed in a circularly polarised transmit/receive head coil. To characterise the position of the eighth cranial nerve and surrounding structures we applied (A) 3D CISS (constructive interference in steady state; figure $1 \mathrm{~A}, \mathrm{C}, \mathrm{D}$ ), and (B) 3D TOF (time of flight; figure 1B) sequences. The CISS sequence is a heavily T2-weighted sequence with steady-state free precession compensating the flow of the cerebrospinal fluid (CSF). Alternating and non-alternating radio frequency pulses are combined in a resulting image displaying a very high contrast between CSF and nervous structures with the benefit of a combination of high signal levels and high spatial resolution. ${ }^{16}{ }^{17}$ (A) 3D CISS-scanparameters: repetition time $(\mathrm{TR})=12.25 \mathrm{~ms} / \mathrm{echo}$ time (TE) $=5.90 \mathrm{~ms}, 70^{\circ}$ flip angle, $180 \times 180 \mathrm{~mm}$ (read $\times$ phase encode) field of view, $64.0 \mathrm{~mm}$ slab thickness, $512 \times 448$ matrix, $1063 \mathrm{D}$ partitions, one slab, $0.35 \times 0.40 \mathrm{~mm}$ pixel size, $0.8 \mathrm{~mm}$ effective section thickness, one acquisition. (B) 3D TOF-scan parameters: Image acquisition before and $3 \mathrm{~min}$ after the administration of $0.1 \mathrm{mmol} / \mathrm{kg}$ body weight of gadopentetate dimeglumine; repetition time $(\mathrm{TR})=21 \mathrm{~ms} / \mathrm{echo}$ time $(\mathrm{TE})=7 \mathrm{~ms}, 20^{\circ}$ flip angle, $200 \mathrm{~mm}$ field of view, $50 \mathrm{~mm}$ slab thickness, $512 \times 384$ matrix, $503 \mathrm{D}$ partitions, one slab, $0.39 \times 0.52 \mathrm{~mm}$ pixel size, $0.8 \mathrm{~mm}$ effective section thickness.

\section{Image analysis}

Reconstructions in transversal (figure 1A), coronal (figure 1C) and sagittal (figure 1D) planes were performed to a slice thickness of $0.8 \mathrm{~mm}$ from the high-resolution 3D CISS sequences using Convis Professional Software on an Intel Icore VII workstation. Therefore, the images were reviewed in their magnetisation

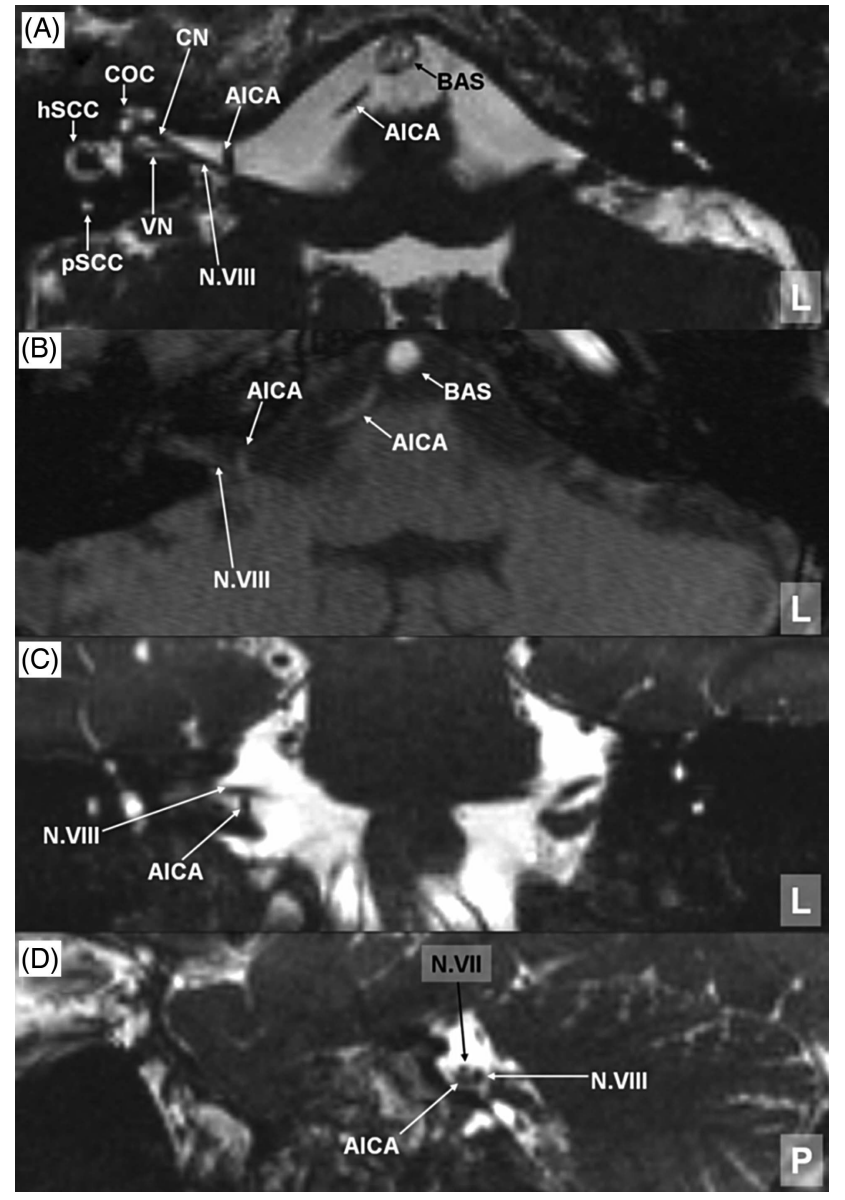

Figure 1 Typical illustration of a neurovascular compression (NVC) of the eighth cranial nerve and the anterior inferior cerebellar artery (AICA). (A): High-resolution constructive interference in steady state (CISS) sequences presenting the NVC between the AICA and the right N.VIII. On this image, the basilar artery (BAS), the cochlea (COC), the posterior semicircular canal (pSCC) as well as the horizontal semicircular canal (hSCC) are also depicted. Additionally, the deviation of the eighth cranial nerve into the $\mathrm{COC}$ nerve $(\mathrm{CN})$ and the vestibular nerve can be seen. (B): On the time-of-flight sequences without gadolinium enhancement, the vessel can be identified as artery, due to the hyperintensity as well as by retracing it to the parent vessel, the BAS. (C): Coronal reconstruction from the CISS sequences, displaying the NVC between the AICA and N.VIII. (D): Sagittal reconstruction with illustration of the close relation between N.VIII, N.VII and the AICA, resulting in a NVC of the eighth cranial nerve.

prepared rapid gradient echo (MPR) sequences in transversal, sagittal and coronal planes, always displaying the corresponding planes in real-time coregistration. The examiners were blinded before analysing the images. All images were assessed by two neurologists $(\mathrm{CB}, \mathrm{TI})$ and by two neuroradiologists (JG, WM-F).

\section{Neurovascular contact}

The absence of a detectable layer of CSF between the eighth cranial nerve and any surrounding blood vessel was defined as NVC. ${ }^{18}$ All identified vessels were followed to their origin and classified anatomically. The differentiation of arterial and venous vessels was made on the basis of two aspects: (A) if the vessel could be retraced to a major arterial (venous) vessel it was classified to be an artery (vein); (B) if the vessel appeared hyperintense in the contrast-enhanced TOF sequences and lacked hyperintensity in the unenhanced TOF sequences, it was 
classified as a vein. Hyperintense presentation of a vessel on enhanced and unenhanced TOF sequences classified a vessel as arterial vessel.

To obtain values of sensitivity and specificity for the MRI procedures, VP patients were diagnosed based on the clinical diagnostic criteria without the imaging results.

\section{Clinical examination}

The patients underwent a detailed medical history, a systematic clinical neurological examination and a detailed neuro-otological and neuro-orthoptic examination, including positioning manoeuvres, stepping test for imbalance in stance and gait, examination with Frenzel's glasses for spontaneous nystagmus, head-shaking test for provocational nystagmus, head impulse test for pathological vestibulo-ocular reflex, screening for central ocular motor disorders by testing smooth pursuit, saccades and the fixation suppression of the vestibulo-ocular reflex.

\section{Neurophysiological testing}

Otolith function was tested by determining tilts of the subjective visual vertical (SVV) and pathological ocular torsion by fundus photographs (for methods, see: ref. 19). Additionally, a binocular 3D video-oculography including rotatory and caloric testing as well as a quantitative eye movement analysis (saccades, smooth pursuit, gaze-evoked nystagmus, optokinetic nystagmus and fixation suppression of the vestibulo-ocular reflex) was performed (for details, see: ref. 20). Cervical vestibular-evoked myogenic potentials (cVEMP) were recorded to test saccular function; amplitude side difference $\geq 50 \%$ was considered as pathological. Bithermal caloric irrigation was performed to determine asymmetry on caloric nystagmus using the formula of Jongkees (side difference $\geq 25 \%$ was considered pathological). ${ }^{21}$ For cochlear function and acoustic processing, acoustically evoked potentials (AEP) were performed.

\section{RESULTS}

\section{Patients}

Twenty VP patients (12 women, 8 men; mean age 47.2 \pm 14.7 years; range: $17-78)$ and $20 \mathrm{TN}$ patients (12 women, 8 men; mean age $48.4 \pm 14.9$ years; range: $25-77)$ were included in our study. A detailed description of the symptomatology of each VP patient with accompanying complaints and signs as well as duration of attacks and the disorder is given in table 1. For the diagnosis of VP, the above mentioned criteria (see box) were applied, but MRI results were excluded from the diagnostic classification to enable the calculation of sensitivity and specificity afterwards. Within the diagnostic category D (additional diagnostic criteria), besides the criterion of a proven NVC in MRI, an alternative criterion is the positive treatment effect, which was present in all the 20 patients. In all included TN patients, the diagnosis was established in accordance with the current guidelines as published by the International Headache Society. $^{22}$ The patients' MR scans were revisited for NVC of the eighth cranial nerve.

\section{Treatment effects in the VP patients}

Eighteen patients were treated with carbamazepine (minimal dose $200 \mathrm{mg} /$ day, maximal dose $600 \mathrm{mg} /$ day), one patient received oxcarbazepine $(600 \mathrm{mg} /$ day $)$ and one patient was treated with gabapentin $(900 \mathrm{mg} /$ day $)$. The patient with gabapentin initially started on carbamazepine and had a full remission on $200 \mathrm{mg} /$ day but developed a skin rash. Also on gabapentin a full relief of symptoms was achieved. The patient with oxcarbazepine also had complete remission of symptoms. From the carbamazepine treated patients three had an incomplete remission with residual symptoms. They described spells of short-lasting unspecific dizziness but were fully happy with the degree of improvement, not willing to increase the dosage or change the regimen of treatment.

Table 1 Clinical symptomatology of patients with vestibular paroxysmia

\begin{tabular}{|c|c|c|c|c|c|}
\hline \multicolumn{6}{|c|}{ Clinical symptomatology } \\
\hline Case & Vertigo & Duration & Provocation & Accompanying & Time \\
\hline 1 & Rotational & Seconds & Head rotation to the right or left & Oscillopsia, gait & 5 years \\
\hline 2 & To-and-fro & $10 \mathrm{~s}$ & Head reclination & Hypacusis, tinnitus, feeling of pressure within left ear & 6 years \\
\hline 3 & Rotational & $4 \mathrm{~s}$ & Head inclination & Oscillopsia & 2 years \\
\hline 4 & Rotational & Seconds & No provocational factors & Oscillopsia, perspiration, gait & 1 year \\
\hline 5 & To-and-fro & Max. $1 \mathrm{~min}$ & No provocational factors & Oscillopsia, nausea, near fainting & 1.5 years \\
\hline 6 & Rotational & Seconds & Head rotation to the left & Oscillopsia, nausea, electrifying sensation left ear & 0.5 years \\
\hline 7 & To-and-fro & Seconds & Head rotation to the left & Gait, tinnitus left ear & 2 years \\
\hline 8 & To-and-fro & Seconds & Head rotation to the right & Gait & 3 years \\
\hline 9 & To-and-fro & Seconds & Head rotation to the left or right & Oscillopsia, gait, nausea & 4.5 years \\
\hline 10 & Rotational & Seconds & Head position extreme left rotated & Oscillopsia & 8 years \\
\hline 11 & Rotational & Seconds & Head rotation to the left & Oscillopsia & 3 months \\
\hline 12 & Rotational & Seconds & Head inclination & Oscillopsia, drop attacks & 4.5 years \\
\hline 13 & Rotational & Max. 1 min & No provocational factors & Oscillopsia, gait & 0.5 years \\
\hline 14 & Rotational & $1-2 \mathrm{~s}$ & Head rotation to the left & Oscillopsia, hypacusis right ear & 1.5 years \\
\hline 15 & Rotational & Seconds & No provocational factors & Oscillopsia & 3.5 years \\
\hline 16 & Rotational & Seconds & No provocational factors & Oscillopsia, gait & 2 years \\
\hline 17 & Rotational & Seconds & No provocational factors & Oscillopsia, nausea & 20 years \\
\hline 18 & Rotational & $1-2 \mathrm{~s}$ & Head rotation to the left or right & Oscillopsia, nausea, vomiting & 1.5 years \\
\hline 19 & To-and-fro & Seconds & Head rotation to the left & Oscillopsia, tinnitus left ear & 1 month \\
\hline 20 & Rotational & Max. 2 min & No provocational factors & Oscillopsia, gait & 3 years \\
\hline
\end{tabular}

The category, 'vertigo' differentiates between rotational and to-and-fro sensations. Duration gives the duration of the isolated vertigo attacks. In the category, 'provocation' provocational factors are displayed as reported by the patients. Accompanying depicts a description of accompanying symptoms and complaints; the category 'gait' presents disturbances of stance and gait. The last category, time, gives the approximated duration since the onset of the vertigo complaints. 
MRI

VP patients

In all 20 VP patients, a NVC between N.VIII and a blood vessel was detected. In the majority of cases $(75 \% ; n=15)$ a contact was documented between the nerve and the anterior inferior cerebellar artery (AICA; figure 1A-D), the posterior inferior cerebellar artery (PICA) in one patient and the vertebral artery (VA) directly at the REZ in two patients. In two patients, the NVC was between a vein and the nerve; in two other patients bilateral neurovascular contacts were revealed. In two patients, a double contact within one nerve could be detected. The mean distance between the REZ and the NVC was $4.8 \mathrm{~mm} \pm 3.3 \mathrm{~mm}$ (range: $0.0-10.2 \mathrm{~mm}$ ), the mean diameter of the compressing vessels was $1.4 \mathrm{~mm} \pm 1.0 \mathrm{~mm}$.

\section{TN patients}

In the control group (20 TN patients without vestibulo-cochlear symptoms), a total of seven (35\%) NVC between the N.VIII and a blood vessel could be detected. In five patients, the compressing vessel was found to be the AICA, in one patient the PICA and in one patient a venous vessel. Mean distance between the REZ and the NVC in the control group was $5.1 \mathrm{~mm} \pm 3.7 \mathrm{~mm}$ (range: $0.0-9.5 \mathrm{~mm}$ ), the mean diameter of the compressing vessels was $1.3 \mathrm{~mm} \pm 0.5 \mathrm{~mm}$ (for details see table 2).

The distribution of the distance between the NVC and the REZ for the groups of VP and TN patients was displayed in a boxplot and a Mann-Whitney-Wilcoxon test was calculated showing no significant difference between the two groups (figure $2 ; \mathrm{p}=0.317$ ).

\section{SENSITIVITY AND SPECIFICITY OF THE MRI PROTOCOL}

A total of 40 patients were examined. In the VP group, all 20 patients fulfilled the clinical diagnostic criteria of VP which were defined as 'gold standard' method for the calculations of sensitivity and specificity. All the VP patients had a positive NVC on MRI, which was defined as 'new' test outcome. Therefore, in the VP group, 20 true positive test results were obtained. As no false negative patients were identified within this group, the sensitivity for MRI compared with the clinical diagnostic criteria (gold standard) was 100\% (20/20). The 95\% binomial proportion CI of sensitivity as calculated by a Wilson score interval was 0.84 to 1.0 . Of the $20 \mathrm{TN}$ patients not fulfilling the clinical diagnostic VP criteria, seven patients (false positive) had a NVC on MRI and 13 patients (true negative) showed no NVC, resulting in a specificity of $65 \%(13 / 20)$. The $95 \%$ binomial proportion CI of specificity as calculated by a Wilson score interval was 0.43 to 0.82 .

\section{CLINICAL AND NEUROPHYSIOLOGICAL RESULTS}

A detailed description of the clinical and neurophysiological results is given in table 3 . Interestingly, in only five patients $(25 \%)$, the neurophysiological testing was unremarkable (cases $3,6,7,8,14)$. Nine patients $(45 \%)$ showed a clear pattern of unilateral loss/reduction of audiovestibular function identifying the affected side (cases 4, 5, 9, 10, 11, 12, 16, 17, 20). The other six patients $(30 \%$; cases $1,2,13,15,18,19)$ revealed complex neurophysiological patterns representing decreased as well as increased nerve function, that is, a combination of nerve lesion and nerve irrigation within the same side. For example, in case 1 with a NVC on the right side, the clinical and neurophysiological testing revealed a provocational nystagmus to the
Table 2 Neurovascular compression (NVC) of the eighth cranial nerve in vestibular paroxysmia and trigeminal neuralgia

Characteristics of the NVC

Patients with vestibular paroxysmia

\begin{tabular}{lllll}
\hline Case & Side of NVC & Vessel & $\begin{array}{l}\text { Distance } \\
\text { to PE (mm) }\end{array}$ & $\begin{array}{l}\text { Vessel } \\
\text { diameter }(\mathrm{mm})\end{array}$ \\
\hline 1 & $\mathrm{R}$ & Venous & 4.2 & 1.4 \\
2 & $\mathrm{R}$ & AICA & 7.8 & 1.1 \\
3 & $\mathrm{R}$ & AICA & 4.3 & 1.2 \\
& $\mathrm{~L}$ & AICA & 1.7 & 0.7 \\
4 & $\mathrm{~L}$ & PICA & 0 & 0.8 \\
5 & $\mathrm{~L}$ & AICA & 4.6 & 1.0 \\
6 & $\mathrm{~L}$ & AICA & 0 & 1.3 \\
7 & $\mathrm{~L}$ & AICA & 9.0 & 1.0 \\
8 & $\mathrm{~L}$ & AICA & 10.2 & 1.3 \\
& $\mathrm{~L}$ & AICA & 0 & 1.3 \\
9 & $\mathrm{~L}$ & AICA & 2.8 & 1.4 \\
10 & $\mathrm{~L}$ & AICA & 6.7 & 1.2 \\
11 & $\mathrm{R}$ & Venous & 3.7 & 1.4 \\
12 & $\mathrm{R}$ & AICA & 7.8 & 1.1 \\
& $\mathrm{R}$ & AICA & 2.5 & 1.1 \\
13 & $\mathrm{R}$ & VA & 0 & 5.3 \\
14 & $\mathrm{R}$ & AICA & 9 & 1.7 \\
15 & $\mathrm{R}$ & AICA & 7.3 & 1.3 \\
16 & $\mathrm{R}$ & AICA & 3.6 & 0.9 \\
& $\mathrm{~L}$ & VA & 6.0 & 3.6 \\
17 & $\mathrm{R}$ & AICA & 0.8 & 0.6 \\
18 & $\mathrm{R}$ & AICA & 6.7 & 1.0 \\
19 & $\mathrm{~L}$ & AICA & 8.1 & 1.1 \\
20 & $\mathrm{~L}$ & AICA & 9.3 & 1.1 \\
$\mathrm{Pati}$ & With & &
\end{tabular}

Patients with trigeminal neuralgia

\begin{tabular}{|c|c|c|c|c|}
\hline Case & $\begin{array}{l}\text { Side of NVC } \\
\text { (of eighth } \\
\text { cranial nerve) }\end{array}$ & Vessel & $\begin{array}{l}\text { Distance } \\
\text { to PE (mm) }\end{array}$ & $\begin{array}{l}\text { Vessel } \\
\text { diameter }(\mathrm{mm})\end{array}$ \\
\hline 1 & n.c. & - & - & - \\
\hline 2 & $\mathrm{R}$ & AICA & 3.8 & 1.5 \\
\hline 3 & n.c. & - & - & - \\
\hline 4 & $\mathrm{R}$ & AICA & 9.4 & 0.7 \\
\hline 5 & n.c. & - & - & - \\
\hline 6 & n.c. & - & - & - \\
\hline 7 & n.c. & - & - & - \\
\hline 8 & n.c. & - & - & - \\
\hline 9 & n.c. & - & - & - \\
\hline 10 & $\mathrm{R}$ & Venous & 1.6 & 1.0 \\
\hline 11 & $\mathrm{~L}$ & AICA & 7.3 & 1.4 \\
\hline 12 & n.c. & - & - & - \\
\hline 13 & n.c. & - & - & - \\
\hline 14 & $\mathrm{~L}$ & PICA & 0 & 2.3 \\
\hline 15 & n.c. & - & - & - \\
\hline 16 & $\mathrm{~L}$ & AICA & 9.5 & 0.9 \\
\hline 17 & n.c. & - & - & - \\
\hline 18 & n.c. & - & - & - \\
\hline 19 & $\mathrm{R}$ & AICA & 3.8 & 1.2 \\
\hline 20 & n.c. & - & - & - \\
\hline \multicolumn{5}{|c|}{$\begin{array}{l}\text { The side of contact (R, right; } L \text {, left) is given as well as the distance to the point of } \\
\text { entry (PE). The category, vessel, defines the exact compressing vessel: AICA, anterior } \\
\text { inferior cerebellar artery; PICA, posterior inferior cerebellar artery; VA, vertebral artery; } \\
\text { venous, venous vessel. In the first part of the table, the characteristics for the NVCs in } \\
\text { the vestibular paroxysmia patients is reported, the second part of the table describes } \\
\text { characteristics of NVC of the eighth nerve in the trigeminal neuralgia patients for } \\
\text { control. } \\
\text { n.c., no neurovascular compression. }\end{array}$} \\
\hline
\end{tabular}




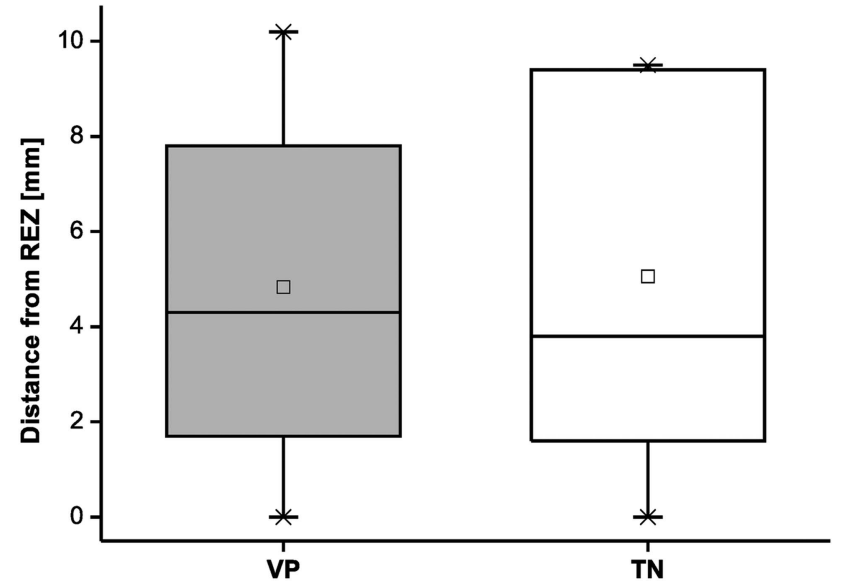

Figure 2 Boxplot illustration of the distance distribution between the neurovascular compression (eighth cranial nerve) and the root-entryzone in patients with vestibular paroxysmia (VP) and patients with trigeminal neuralgia (TN). The boxes include the second and third quartiles, the line within the box displaying the median, the square within the box characterising the mean and the whiskers with the cross-marks showing maximum and minimum values. As tested by a Mann-Whitney-Wilcoxon test the distribution did not differ significantly $(p=0.317)$.

left (compatible with a right-sided lesion or a left-sided irrigation) and a pathological caloric side difference of 32\% in favour of the right side which represent signs of hypofunction and excitation of the right N.VIII. In case 2, a right-sided NVC resulted in a provocational nystagmus to left (compatible with a right-sided lesion), a pathological caloric side difference of $41 \%$ in favour of the right side, a pathological ocular torsion of both eyes to the left, and pathological tilts of SVV to the left (both compatible with a lesion left or an irrigation right), and finally a right-sided pathological AEP. Thus, the testing disclosed signs of a hypofunction and an excitation of the right N.VIII (for details, see table 3).

\section{DISCUSSION}

NVC of the eighth cranial nerve was detected with a sensitivity of $100 \%$ in our VP patients and a specificity of $65 \%$ in our TN control group by a standardised MRI protocol. We were able to demonstrate that VP can be elicited by a NVC of the eighth cranial nerve by any type and size of compressing blood vessel along the whole intracisternal segment with a length of more than $10 \mathrm{~mm}$ of the eighth cranial nerve. A contact could be assigned to the AICA in $75 \%$, the VA in $10 \%$, the PICA in 5\%, and a vein in another $10 \%$. Furthermore, the broad range of the symptoms could also be comprehended, as the neurophysiological test results presented three types of pathology: first, isolated functional deficits, second, combined deficits, and third, at first glance contradicting combinations of audiovestibular deficits (hypoexcitability) and hyperexcitability occurring in the same nerve. The latter complex pattern represents the simultaneous combination of lesion and irrigation of the eighth cranial nerve which was described earlier in single cases. ${ }^{23} 24$

Table 3 Clinical and neurophysiological parameters in vestibular paroxysmia

\begin{tabular}{|c|c|c|c|c|c|c|c|c|c|}
\hline \multicolumn{10}{|c|}{ Results of clinical and neurophysiological examination } \\
\hline CASE & NVC & VOR & ORTH & VOG CAL & OT & SVV & cVEMP & AEP & LAT \\
\hline 1 & $\mathrm{R}$ & $\mathrm{np}$ & PN L & $R>L \quad 32 \%$ & $\mathrm{np}$ & $\mathrm{np}$ & $\mathrm{np}$ & $\mathrm{np}$ & Cont \\
\hline 2 & $\mathrm{R}$ & $\mathrm{np}$ & PN L & $\mathrm{R}>\mathrm{L} 41 \%$ & $\mathrm{~L}$ & $\mathrm{~L}$ & $\mathrm{np}$ & $\mathrm{R}$ & Cont \\
\hline 3 & L/R & $\mathrm{np}$ & $\mathrm{np}$ & $\mathrm{np}$ & $\mathrm{np}$ & $\mathrm{np}$ & $\mathrm{np}$ & $\mathrm{np}$ & Norm \\
\hline 4 & $\mathrm{~L}$ & $\mathrm{~L}$ & SPN R & $R>L 60 \%$ & $\mathrm{~L}$ & $\mathrm{~L}$ & L DEC & $\mathrm{np}$ & Corr \\
\hline 5 & $\mathrm{~L}$ & $\mathrm{np}$ & $\mathrm{np}$ & $\mathrm{np}$ & $\mathrm{np}$ & $\mathrm{L}$ & $\mathrm{np}$ & $\mathrm{L}$ & Corr \\
\hline 6 & $\mathrm{~L}$ & $\mathrm{np}$ & $\mathrm{np}$ & $\mathrm{np}$ & $\mathrm{np}$ & $\mathrm{np}$ & $\mathrm{np}$ & $\mathrm{np}$ & Norm \\
\hline 7 & $\mathrm{~L}$ & $\mathrm{np}$ & $\mathrm{np}$ & $\mathrm{np}$ & $\mathrm{np}$ & $\mathrm{np}$ & $\mathrm{np}$ & $\mathrm{np}$ & Norm \\
\hline 8 & $\mathrm{~L} / \mathrm{L}$ & $\mathrm{np}$ & $\mathrm{np}$ & $\mathrm{np}$ & $\mathrm{np}$ & $\mathrm{np}$ & $\mathrm{np}$ & $\mathrm{np}$ & Norm \\
\hline 9 & $\mathrm{~L}$ & $\mathrm{np}$ & $\mathrm{np}$ & $\mathrm{np}$ & $\mathrm{np}$ & $\mathrm{L}$ & $\mathrm{np}$ & $\mathrm{np}$ & Corr \\
\hline 10 & $\mathrm{~L}$ & $\mathrm{np}$ & USPN & $\mathrm{np}$ & $\mathrm{np}$ & $\mathrm{L}$ & $\mathrm{np}$ & $\mathrm{np}$ & Corr \\
\hline 11 & $\mathrm{R}$ & $\mathrm{np}$ & $\mathrm{np}$ & $\mathrm{R}<\mathrm{L} 24 \%$ & $\mathrm{np}$ & $\mathrm{np}$ & $\mathrm{np}$ & $\mathrm{R}$ & Corr \\
\hline 12 & $R / R$ & $\mathrm{np}$ & $\mathrm{np}$ & $\mathrm{R}<\mathrm{L} 28 \%$ & $\mathrm{np}$ & $\mathrm{np}$ & R DEC & $\mathrm{np}$ & Corr \\
\hline 13 & $\mathrm{R}$ & $\mathrm{np}$ & $\mathrm{np}$ & $R>L 55 \%$ & $\mathrm{np}$ & $\mathrm{np}$ & $\mathrm{np}$ & $\mathrm{R}$ & Cont \\
\hline 14 & $\mathrm{R}$ & $\mathrm{np}$ & $\mathrm{np}$ & $\mathrm{np}$ & $\mathrm{np}$ & $\mathrm{np}$ & $\mathrm{np}$ & $\mathrm{np}$ & Norm \\
\hline 15 & $\mathrm{R}$ & $\mathrm{L}$ & PN L & $R<L \quad 100 \%$ & $\mathrm{np}$ & $\mathrm{L}$ & $\mathrm{np}$ & $\mathrm{np}$ & Cont \\
\hline 16 & $\mathrm{~L} / \mathrm{R}$ & $\mathrm{np}$ & PN L & $\mathrm{np}$ & $\mathrm{np}$ & $\mathrm{np}$ & $\mathrm{np}$ & $\mathrm{np}$ & Corr \\
\hline 17 & $\mathrm{R}$ & $\mathrm{np}$ & $\mathrm{np}$ & $\mathrm{np}$ & $\mathrm{np}$ & $\mathrm{np}$ & R DEC & $\mathrm{np}$ & Corr \\
\hline 18 & $\mathrm{R}$ & $\mathrm{np}$ & $\mathrm{np}$ & $\mathrm{np}$ & $\mathrm{np}$ & $\mathrm{np}$ & L DEC & $\mathrm{np}$ & Cont \\
\hline 19 & $\mathrm{~L}$ & $\mathrm{np}$ & $\mathrm{np}$ & $\mathrm{R}<\mathrm{L} 67 \%$ & $\mathrm{np}$ & $\mathrm{L}$ & R DEC & $\mathrm{np}$ & Cont \\
\hline 20 & $\mathrm{R}$ & $\mathrm{np}$ & $\mathrm{np}$ & $\mathrm{R}<\mathrm{L} 38 \%$ & $\mathrm{np}$ & $\mathrm{R}$ & $\mathrm{np}$ & $\mathrm{np}$ & Corr \\
\hline
\end{tabular}


The site of NVC along the eighth cranial nerve

The data on standardised MRI analyses of NVC syndromes of the eighth cranial nerve is sparse. In a recent MRI and neurophysiological study on 34 patients suspected to have a NVC syndrome, a $100 \%$ prevalence of contact between the AICA and the eighth cranial nerve was detected. ${ }^{15}$ Since a positive NVC was a prerequisite for study entry, the study does not help in respect to the differential diagnosis of VP. The exact site of the NVC, however, has not been described. We were able to proof a NVC in all 20 patients with the clinical syndrome of VP (sensitivity $100 \%$; specificity $65 \%$ ). While the sensitivity in the VP patients with $100 \%$ was very good, the specificity of $65 \%$ in the TN group was rather moderate. As no audiovestibular testing was performed on the TN patients, it cannot be decided if some of the 'false positive' NVC of N.VIII in the TN patients had an audiovestibular correlate. Thus, the specificity could possibly be a little higher than $65 \%$. Our results, however, correspond to previous findings in $22 \mathrm{VP}$ patients who underwent MRI scans including CISS sequences; in all but one patient, a NVC was found. ${ }^{3}$ An exact MRI analysis (distance to PE, diameter of vessel) of the NVC was not presented.

Though the predictive value of high-resolution MRI (including CISS sequences) in identifying the exact site of NVC was not directly assessed in this study, one might infer from the high sensitivity of $100 \%$ (and moderate specificity of $65 \%$ ) that MRI should be appropriate to exclude VP in uncertain cases or to diagnose differential diseases, such as vestibular schwannoma or tumours within the cerebellopontine angle. Furthermore, the exact characterisation of the NVC and the side of the contact by MRI is helpful for the interpretation of ambiguous results in clinical and neurophysiological examination-at least in cases of unilateral NVC.

\section{Which part of the nerve is especially sensitive to a NVC?}

Ryu and colleagues performed posterior fossa surgery and neurovascular decompression of the fifth as well as the eighth cranial nerve and documented the sites of the NVC. They found it to be along the whole intracisternal segment of the eighth cranial nerve. ${ }^{25} 26$ These results are in accordance with our results, as we found NVC along the whole intracisternal part of the nerve starting from $0 \mathrm{~mm}$ up to $10.2 \mathrm{~mm}$ close to the internal acoustic porus. These data fit to a postmortem study on the anatomy of the cranial nerves by Lang: the transition zone, where oligodendrocytes change into Schwann cells, ranges from $6 \mathrm{~mm}$ to $15 \mathrm{~mm}$ as measured from the PE. ${ }^{27}$ Most often, this transition zone was found in the area of the internal acoustic porus. Thus, the largest part of the intracisternal range of the eighth cranial nerve is covered by central myelin and obviously oligodendrocytes seem to be particular vulnerable for deficits induced by NVC. Interestingly, the intracisternal part of central myelin is shorter in other cranial nerves except for the facial nerve. ${ }^{27}$ Therefore, the eighth cranial nerve appears to be specifically susceptible for a compression-induced dysfunction due to the long distance covered by central myelin. Particularly in the light of the variety of isolated and combined vestibular as well as auditory symptoms, the importance and benefit of a standardised diagnostic procedure in patients suspected for VP should be underlined. The application of high-resolution MRI including CISS sequences due to the high sensitivity should be implemented to exclude VP in unclear cases, or to identify differential diseases, for example, vestibular schwannomas or other tumours of the cerebellopontine angle.
Variations of the audiovestibular findings

There are only few studies with detailed descriptions of the audiovestibular testing. In a recent study on patients meeting the radiological criteria for a NVC of the eighth nerve, an extremely high prevalence of pathological audiovestibular testing was reported. ${ }^{15}$ However, some uncertainties in the interpretation of these data have to be taken into consideration. No correlation between the detected NVC and the pathological neurophysiological findings was performed and it appears unclear how a peripheral vestibular nerve deficit is capable of inducing a pathological optokinetic nystagmus, a sign of central dysfunction. The study by Hüfner and co-workers gave a detailed description of a comprehensive audiovestibular testing. Over a time course of 5.7 years, a decrease in pathological gaze-evoked nystagmus, an increase in pathological findings on electronystagmography, ocular torsion and static SVV results were found. ${ }^{3}$ A direct correlation between the pathological findings on audiovestibular testing and the NVC in MRI could not be performed since in $42 \%$ of the patients a bilateral contact was present. ${ }^{3}$ This prevalence of pathological audiovestibular test results roughly matched the numbers of our patient collective. In older studies, it was already described that vestibular test results can be contradicting and of limited localising value, whereas pathological findings in auditory testing were meant to be of high localising value. ${ }^{28}$ On the other hand, other studies were not able to discover any specific results, and even revealed normal findings in the majority of tests and patients, or they found in about $30 \%$ only a slightly reduced caloric responsiveness. ${ }^{29}{ }^{30}$ However, the significance of audiovestibular findings in the older studies was limited as a more detailed testing of vestibular functions, such as otolith function, for example, by cVEMPs and measurements of ocular torsion and SVV, was not available. Such a detailed vestibular testing to date identified six of our patients with signs of excitation as well as hypofunction within the same nerve. We therefore conclude that an asymmetry of caloric responsive nystagmus as sign of canal paresis in some cases has to be interpreted as an increased excitability of the vestibular nerve/nuclei on one side and not as 'hyporesponsiveness' of the contralateral side. In addition to the neurophysiological audiovestibular test results, hyperventilation-induced nystagmus as the most sensitive clinical sign of VP was not tested in all our patients. Therefore, it was not included in the analyses. Future studies should, in addition to the procedures applied in our study, systematically evaluate hyperventilation-induced nystagmus.

\section{Pathophysiology of NVC}

While NVC syndromes have been reported for several cranial nerves, and effective treatment regimens were established-of invasive as well as non-invasive nature-the pathophysiology remains controversial. ${ }^{7}$ On the one hand a 'peripheral hypothesis' postulating the local compression and with it the peripheral nerve pathology as causative. ${ }^{31-33}$ On the other hand a 'central hypothesis' defines a dysfunction within the cranial nerve nuclei with increased excitability or even a dysfunction at the level of thalamic-cortical projections as well as on cortical level itself as causal. $^{34}{ }^{35}$ In the context of the pathophysiological discussion, Adams even postulated, that NVC is not correlated with a patient's symptoms at all. ${ }^{36}$ In conclusion, a 'dual pathology' yet seems to be the most likely assumption on the various concepts and arguments: The peripheral NVC seems to be a necessary factor triggering the development of symptoms defining the clinical picture of a NVC syndrome. In addition to the peripheral compression, a central pathology inducing hyperexcitability 
within the cranial nerve nuclei, or even a reduced inhibitory projection to the cranial nerve nuclei, seems to be mandatory. Furthermore, additional factors must play a role in the development of the clinical picture of NVC syndromes, as the presence of a nerve-vessel contact alone does not suffice in triggering the disease. Such yet unknown factors might be altered membrane potentials or latent nerve injuries. ${ }^{7}$ Data on the pathophysiological concept of NVC syndromes arises mainly from studies on the fifth and the seventh cranial nerve. The data on such phenomena for the eighth cranial nerve is sparse.

While central sensitisation and decreased central inhibitory activity both resulting in hyperexcitability were already described in the context of symptom development for the fifth cranial nerve, a systematical evaluation of such mechanisms for the eighth nerve is lacking entirely. ${ }^{37} 38$ There is only one report on a patient with alternating episodes of vestibular nerve excitation and failure due to an arachnoid cyst in the right cerebellopontine angle that distorted the vestibulo-cochlear nerve. ${ }^{24}$ The most likely cause was a transition from a conduction block to ectopic discharges that occurs when various peripheral nerves are compressed. Animal experiments have shown that longlasting compression of a nerve leads to depolarisation of axons and conduction block due to direct nerve compression or ischaemia caused by compression of nerve vessels. ${ }^{39}$ Moreover, short-duration mechanical compression of an ischaemic depolarised nerve induces ectopic firing. ${ }^{40}$ Both mechanisms explain the signs of hypoexcitability and hyperexcitability within the same nerve. In the context of the pathophysiological discussion in NVC syndromes, our data provide the first systematic evidence which supports the idea of a dual pathology for a NVC of the eighth cranial nerve. Besides the picture of peripheral nerve pathology with a functional deficit as depicted by the corresponding electrophysiological procedures, we also found distinct signs of an increased excitability. Our data give evidence that such phenomena play a relevant role (30\% of the cases) in the symptomatology of VP. At least, the presence of a NVC has to be a prerequisite, or precondition, for the further development of the full clinical VP picture. Thus, only in the context of imaging and neurophysiological results the complex picture of VP can be unambiguously classified.

\section{CONCLUSION}

Due to the long intracisternal course with central myelin, the eighth cranial nerve is highly susceptible to a NVC by any surrounding artery and vein inducing the clinical presentation of VP. Applying a combination of clinical and neurophysiological diagnostic procedures as well as standardised high-resolution MRI with 3D CISS sequences, the diagnostic certainty is very high. The combination of the different methods in the patients suspected for VP is mandatory. Especially, due to the moderate specificity of MRI on its own, MRI should not be applied to diagnose NVC. In the context of suspected VP, MRI is capable of (1) excluding cases without a NVC, (2) excluding differential diagnosis and (3) to define the side of pathology in cases of contradicting neurophysiological and clinical test results. Detailed neurophysiological audiovestibular testing is capable of depicting deficit syndromes and also nerve excitation and/or central sensitisation with consecutive hyper-responsiveness of the vestibular afferents. In conclusion, the combination of neurophysiological and imaging techniques is recommended for patients suspected with VP to safely classify the side and degree of damage, and to finally have a better control of treatment success in the follow-up of the patients.
Acknowledgements We would like to thank Anja Schröer for expert technical help and Judy Benson for desktop editing the manuscript. This work contains essential parts of the doctoral thesis of Tugba Ibis.

Contributors CB designed the study, assisted in the acquisition of the MRI data, analysed and interpreted all results and wrote the manuscript. JG programmed the standardised MRI procedures, conducted all MRI measurements, analysed the results and critical revised the manuscript. HHK helped in the analyses and interpretation of the results, assisted in the writing of the manuscript and critically revised the manuscript. FT assisted in planning and designing the study, supervised the study and critically revised the various versions of the manuscript. TI analysed all MRI images, assisted in the processing and interpretation of the results and assisted in the writing of the manuscript. WM-F assisted in planning and designing the study, took part in the planning of MRI procedures, analysed the results and critically revised the manuscript. MD designed the study, supervised the study, analysed and interpreted the results, assisted in the writing of the manuscript and revised the various versions of the manuscript.

Funding This work was supported by BMBF Grant 01 GW 0642 of the German Ministry of Education and Research and the German Center for Vertigo and Balance Disorders-IFBLMU.

\section{Competing interests None.}

Ethics approval The study was approved by the ethics committee of the medical chamber in Rhineland-Palatinate.

Provenance and peer review Not commissioned; externally peer reviewed.

\section{REFERENCES}

1 Brandt T, Dieterich M. VIIIth nerve vascular compression syndrome: vestibular paroxysmia. Baillieres Clin Neurol 1994;3:565-75

2 Brandt T, Dieterich M. Vestibular paroxysmia: vascular compression of the eighth nerve? Lancet 1994;343:798-9.

3 Hüfner $K$, Barresi D, Glaser $M$, et al. Vestibular paroxysmia: diagnostic features and medical treatment. Neurology 2008;71:1006-14.

4 Jannetta PJ. Neurovascular cross-compression in patients with hyperactive dysfunction symptoms of the eighth cranial nerve. Surg Forum 1975;26:467-9.

5 Jannetta PJ. Microsurgery of cranial nerve cross-compression. Clin Neurosurg 1979;26:607-15

6 Jannetta PJ, Moller MB, Moller AR. Disabling positional vertigo. N Engl J Med 1984:310:1700-5.

7 Møller AR. Vascular compression of cranial nerves: II: pathophysiology. Neurol Res 1999:21:439-43.

8 De Ridder $\mathrm{D}$, Moller A, Verlooy J, et al. Is the root entry/exit zone important in microvascular compression syndromes?. Neurosurgery 2002;51:427-33.

9 Wilkins RH. Neurovascular compression syndromes. Neurol Clin 1985;3:359-72.

10 Afifi AK, Bell WE, Bale JF, et al. Recurrent lateral rectus palsy in childhood. Pediatr Neurol 1990;6:315-18.

11 Ohtsuka K, Sone A, Igarashi Y, et al. Vascular compressive abducens nerve palsy disclosed by magnetic resonance imaging. Am J Ophthalmol 1996:122:416-19.

12 Yousry I, Dieterich M, Naidich TP, et al. Superior oblique myokymia: magnetic resonance imaging support for the neurovascular compression hypothesis. Ann Neurol 2002;51:361-8.

13 Sandvand KA, Ringstad G, Kerty E. Periodic abducens nerve palsy in adults caused by neurovascular compression. J Neurol Neurosurg Psychiatry 2008;79:100-2.

14 Sun X, Liang C, Liu C, et al. Oculomotor paralysis: 3D-CISS MR imaging with MPR in the evaluation of neuralgic manifestation and the adjacent structures. Eur $J$ Radiol 2008;73:221-3

15 Markowski J, Gierek T, Kluczewska E, et al. Assessment of vestibulocochlear organ function in patients meeting radiologic criteria of vascular compression syndrome of vestibulocochlear nerve-diagnosis of disabling positional vertigo. Med Sci Monit 2011;17:CR169-73.

16 Casselman JW, Kuhweide R, Dehaene I, et al. Magnetic resonance examination of the inner ear and cerebellopontine angle in patients with vertigo and/or abnormal findings at vestibular testing. Acta Otolaryngol Supp/ 1994;513:15-27.

17 Rabinov JD, Barker FG, McKenna MJ, et al. Virtual cisternoscopy: 3D MRI models of the cerebellopontine angle for lesions related to the cranial nerves. Skull Base 2004;14:93-9; discussion 99.

18 Yousry I, Camelio S, Wiesmann $\mathrm{M}$, et al. Detailed magnetic resonance imaging anatomy of the cisternal segment of the abducent nerve: Dorello's canal and neurovascular relationships and landmarks. J Neurosurg 1999;91:276-83.

19 Dieterich M, Brandt T. Ocular torsion and tilt of subjective visual vertical are sensitive brainstem signs. Ann Neurol 1993;33:292-9.

20 Best C, Eckhardt-Henn A, Diener G, et al. Interaction of somatoform and vestibular disorders. J Neurol Neurosurg Psychiatry 2006;77:658-64.

21 Honrubia V. Quantitative vestibular function tests and the clinical examination. In: Herdman SJ, ed. Vestibular rehabilitation. Philadelphia: Davis, 1994:113-64.

22 IHS. The International Classification of Headache Disorders: 2nd edition. Cephalalgia 2004;24(SI):9-160. 


\section{Cranial neuropathies}

23 Straube A, Buttner U, Brandt T. Recurrent attacks with skew deviation, torsional nystagmus, and contraction of the left frontalis muscle. Neurology 1994;44:177-8.

24 Arbusow V, Strupp M, Dieterich $M$, et al. Alternating episodes of vestibular nerve excitation and failure. Neurology 1998;51:1480-3.

25 Ryu $\mathrm{H}$, Yamamoto $\mathrm{S}$, Sugiyama $\mathrm{K}$, et al. Neurovascular compression syndrome of the eighth cranial nerve. Can the site of compression explain the symptoms? Acta Neurochir (Wien) 1999;141:495-501.

26 Ryu H, Yamamoto S, Sugiyama K, et al. Neurovascular decompression of the eighth cranial nerve in patients with hemifacial spasm and incidental tinnitus: an alternative way to study tinnitus. J Neurosurg 1998;88:232-6.

27 Lang J. [Anatomy, length and blood vessel relations of "central" and "peripheral" paths of intracisternal cranial nerves]. Zentralb/ Neurochir 1982:43:217-58.

28 Bergsneider M, Becker DP. Vascular compression syndrome of the vestibular nerve: a critical analysis. Otolaryngol Head Neck Surg 1995:112:118-24.

29 Noguchi Y, Ohgaki T, Tsunoda A, et al. [Clinical study in vertiginuous patients suspected of having neurovascular compression syndrome of the eighth cranial nerve]. Nihon Jibiinkoka Gakkai Kaiho 1997;100:492-8.

30 Ryu H, Yamamoto S, Sugiyama K, et al. Neurovascular compression syndrome of the eighth cranial nerve. What are the most reliable diagnostic signs? Acta Neurochir (Wien) 1998;140:1279-86.

31 Gardner WJ. Cross talk-the paradoxical transmission of a nerve impulse. Arch Neurol 1966;14:149-56.
32 Nielsen VK. Pathophysiology of hemifacial spasm: I. Ephaptic transmission and ectopic excitation. Neurology 1984;34:418-26.

33 Granit R, Leksell L, Skoglund CR. Fibre interaction in injured or compressed region of nerve. Brain 1944;67:125-40.

34 Esteban A, Molina-Negro P. Primary hemifacial spasm: a neurophysiological study. J Neurol Neurosurg Psychiatry 1986;49:58-63.

35 Ishikawa M, Ohira T, Namiki J, et al. Electrophysiological investigation of hemifacial spasm after microvascular decompression: $F$ waves of the facial muscles, blink reflexes, and abnormal muscle responses. J Neurosurg 1997;86:654-61.

36 Adams CB. Microvascular compression: an alternative view and hypothesis. J Neurosurg 1989;70:1-12.

37 Fromm GH, Chattha AS, Terrence CF, et al. Role of inhibitory mechanisms in trigeminal neuralgia. Neurology 1981;31:683-7.

38 Obermann M, Yoon MS, Ese D, et al. Impaired trigeminal nociceptive processing in patients with trigeminal neuralgia. Neurology 2007;69:835-41.

39 Dahlin LB, Shyu BC, Danielsen N, et al. Effects of nerve compression or ischaemia on conduction properties of myelinated and non-myelinated nerve fibres. An experimental study in the rabbit common peroneal nerve. Acta Physiol Scand 1989:136:97-105.

40 Sugawara O, Atsuta Y, Iwahara T, et al. The effects of mechanical compression and hypoxia on nerve root and dorsal root ganglia. An analysis of ectopic firing using an in vitro model. Spine (Phila Pa 1976) 1996;21:2089-94. 

paroxysmia: contradiction and correlation

Christoph Best, Joachim Gawehn, Heidrun H Krämer, Frank Thömke, Tugba Ibis, Wibke Müller-Forell and Marianne Dieterich

J Neurol Neurosurg Psychiatry 2013 84: 1349-1356 originally published online September 4, 2013

doi: 10.1136/jnnp-2013-305513

Updated information and services can be found at:

http://jnnp.bmj.com/content/84/12/1349

References

These include:

\title{
Email alerting service
}

This article cites 39 articles, 6 of which you can access for free at: http://jnnp.bmj.com/content/84/12/1349\#BIBL Receive free email alerts when new articles cite this article. Sign up in the
box at the top right corner of the online article.

Topic Articles on similar topics can be found in the following collections

Collections

\author{
Brain stem / cerebellum (629) \\ Cranial nerves (476) \\ Eating disorders (25) \\ Neuromuscular disease (1194) \\ Pain (neurology) (657) \\ Peripheral nerve disease (595) \\ Radiology (1614) \\ Radiology (diagnostics) (1215)
}

\section{Notes}

To request permissions go to:

http://group.bmj.com/group/rights-licensing/permissions

To order reprints go to:

http://journals.bmj.com/cgi/reprintform

To subscribe to BMJ go to:

http://group.bmj.com/subscribe/ 